\title{
Polygon-based contact description for modeling arbitrary polyhedra in the Discrete Element Method
}

\author{
Bart Smeets $^{\mathrm{a}, \mathrm{b}, *}$, Tim Odenthal ${ }^{\mathrm{a}, \mathrm{b}}$, Simon Vanmaercke ${ }^{\mathrm{a}}$, Herman Ramon ${ }^{\mathrm{a}}$ \\ ${ }^{a}$ KU Leuven - MeBioS, Kasteelpark Arenberg 30, 3001 Heverlee \\ ${ }^{b}$ KU Leuven - Biomechanics Section, Celestijnenlaan 300c, 3001 Heverlee
}

\begin{abstract}
Many real-life applications of the Discrete Element Method (DEM) require a particle description which accounts for irregular and arbitrary shapes. In this work, a novel method is presented for calculating contact force interactions between polyhedral particles. A contact between two polyhedra is decomposed as a set of contacts between individual polygonal facets. For each polygon-polygon contact, an individual contact force is obtained by integrating a linear pressure over the area of its intersection. Both convex as well as partially concave polyhedra can be accurately represented. The proposed algorithm is validated by comparing to previously published experimental and computational gravitational particle depositions of identical cubes. Finally, the model is demonstrated in simulations of gravitational packing of various other polyhedral shapes.
\end{abstract}

Keywords: Discrete Element Method, arbitrary shapes, contact model, polyhedra, gravitational packing

\footnotetext{
${ }^{*}$ Corresponding author

Email address: bart.smeets@biw.kuleuven.be, Tel.+3216321478 (Bart Smeets)
} 


\section{Introduction}

In granular assemblies, particle shape has been shown to be a determining parameter affecting, among else, a system's response upon loading [1, 2], packing density, stress patterns [3] and ratcheting behaviour [4]. In the Discrete Element Method (DEM), which tries to describe granular systems as assemblies of distinct, explicitly modeled bodies interacting by means of contact forces [5], particle shape is often approximated using a simplified geometrical representation, e.g. spheres. Many applications, however, require a more elaborate description of irregular bodies.

During the last years, many advances are made in shape description for the Discrete Element Method. Instead of spheres, ellipsoids [6, 7, 8], superquadrics [9, 10], and polyhedra [5, 11] have been used to approximate particle shape. Other approaches use composites of more simple shape primitives, such as spheres [12, 13, 14], ellipsoids [15] and spheropolygons [16]. A variation of DEM, the Granular Element Method (GEM), uses Non-Uniform Rational Basis-Splines (NURBS) to capture grain shape, offering a flexible and robust algorithm to account for arbitrary rounded shapes [17, 18]. Another method for modeling arbitrary rounded shapes is based on triangulated surface meshes in which the local curvature is used for a Hertzian contact force formulation [19].

In this work, we propose a flexible and easy-to-implement algorithm to model irregular polyhedral particles. The presented method represents particles using a surface mesh containing polygonal facets, and formulates contact forces based on individual interactions between two contacting bodies. For each polygon-polygon contact, a linear elastic and dissipative pressure is used which is numerically integrated over the intersection of the two polygons. Because each contact between two polygons is resolved independently, the method benefits from efficient contact detection and can be easily parallelized. In Section 2, it is explained how contact forces can be computed between two arbitrarily shaped polyhedra. Next, in Section 3, the model is validated by comparing to simulations of gravitational deposition of cubes and further demonstrated by showing analogous deposition of various other polyhedral particles. 


\section{Model description}

\subsection{Contact detection}

Contact detection, i.e. the generation of a list of contact candidates, is performed on the level of individual polygonal facets, instead of between two complete polyhedral bodies. Bounding boxes [20] are constructed for each individual polygon. Using these bounding boxes several efficient contact detection methods can be applied, such as (multi-) grid [21, 22, 23, 24] and octree [25, 26] methods.

For each set of two polygons, these algorithms can cheaply determine whether or not their bounding boxes are overlapping, and are therefore likely to have physical contact. With these contact detection methods, the computational effort does not scale with the number of polygons being used in the simulation, but only with the number of polygons that are actually in contact (see section 3.4 and [19]).

\subsection{Geometrical contact properties}

Contact pressures are calculated on the contact plane between two polygons $P_{1}$ and $P_{2}$ with normal vectors $\hat{\mathbf{n}}_{1}$ and $\hat{\mathbf{n}}_{2}$. For this, an intersection polygon $S_{12}$ is first determined. In the case of equal material properties, the plane in which $S_{12}$ lies is chosen as the bisection of the planes of $P_{1}$ and $P_{2}$. The contact normal unit vector is therefore approximated as:

$$
\hat{\mathbf{n}}_{12}=\frac{\hat{\mathbf{n}}_{2}-\hat{\mathbf{n}}_{1}}{\left\|\hat{\mathbf{n}}_{2}-\hat{\mathbf{n}}_{1}\right\|}
$$

If the two contacting bodies have a different stiffness, the contributions of $\hat{\mathbf{n}}_{1}$ and $\hat{\mathbf{n}}_{2}$ to $\hat{\mathbf{n}}_{12}$ should in principle be inversely weighted with their stiffness.

All three planes characterized by $\hat{\mathbf{n}}_{1}, \hat{\mathbf{n}}_{2}$ and $\hat{\mathbf{n}}_{12}$ contain the plane-plane intersection line defined by the vector $\hat{\mathbf{l}}_{12}=\hat{\mathbf{n}}_{1} \times \hat{\mathbf{n}}_{2}$ and a point $\mathbf{s}$ chosen on the intersection line.

Next, $P_{1}$ and $P_{2}$ are projected on the contact plane along the direction of respectively $\hat{\mathbf{n}}_{2}$ and $\hat{\mathbf{n}}_{1}$, yielding the projections $P_{1}^{\prime}$ and $P_{2}^{\prime}$ (see Fig. 1(a) and Fig. 1(c)]. $S_{12}$ is then obtained by computing the side of the intersection between $P_{1}^{\prime}$ and $P_{2}^{\prime}$ which is in the direction of positive overlap (Fig. 1(b)).

At a given test point $\mathbf{x}$ inside $S_{12}$, the overlap distance $\delta_{12}$ can be calculated as:

$$
\delta_{12}(\mathbf{x})=2 \tan (\alpha)\left[(\mathbf{x}-\mathbf{s}) \cdot\left(\hat{\mathbf{n}}_{12} \times \hat{\mathbf{l}}_{12}\right)\right]
$$


with $\cos (\alpha)=\hat{\mathbf{n}}_{12} \cdot \hat{\mathbf{n}}_{1}$. The contact point $\mathbf{c}$ is approximated as the mean of the corners of $S_{12}$, weighted by their corresponding overlap distance according to Eq. (2).

In every $\mathbf{x} \in S_{12}$, a relative contact velocity is defined as:

$$
\begin{aligned}
\mathbf{v}_{12}(\mathbf{x}) & =\mathbf{v}_{2}^{\text {dof }}-\mathbf{v}_{1}^{\text {dof }} \\
& +\mathbf{w}_{2}^{\text {dof }} \times\left(\mathbf{x}-\mathbf{x}_{2}^{\text {dof }}\right) \\
& -\mathbf{w}_{1}^{\text {dof }} \times\left(\mathbf{x}-\mathbf{x}_{1}^{\text {dof }}\right),
\end{aligned}
$$

where $\mathbf{x}_{i}^{\text {dof }}, \mathbf{v}_{i}^{\text {dof }}$ and $\mathbf{w}_{i}^{\text {dof }}$ are respectively the center of mass position, velocity and angular velocity of the polyhedron to which polygon $P_{i}$ belongs.

To deal with issues of numerical accuracy - e.g. exact flat contacts - or efficiency - e.g. early contact reject cases, additional calculations are performed. These are briefly summarized in 5 .

\subsection{Normal contact force calculation}

The normal elastic contact pressure $p_{n, e}$ at a test point $\mathbf{x}$ increases linearly with the overlap distance:

$$
p_{n, e}(\mathbf{x})=k_{l} \delta_{12}(\mathbf{x}),
$$

with $k_{l}$ the layer stiffness $(\mathrm{Pa} / \mathrm{m})$. For a flat linearly elastic layer with thickness $h$ (see also Appendix 1), $k_{l}$ is related to the bulk modulus $K$ as: $k_{l}=K / h$ 27.

A normal dissipative (damping) pressure is calculated using the normal relative contact velocity:

$$
p_{n, d}(\mathbf{x})=-c_{l}\left(\mathbf{v}_{12}(\mathbf{x}) \cdot \hat{\mathbf{n}}_{12}\right),
$$

with $c_{l}$ a layer damping coefficient $\left(\mathrm{kg} /\left(\mathrm{m}^{2} \mathrm{~s}\right)\right)$.

The intersection polygon $S_{12}$ is either already a triangle or can be trivially subdivided into $N_{S}$ triangles by connecting one corner point with all edges. The contact force between polygons $P_{1}$ and $P_{2}$ is obtained by integrating the normal pressure over $S_{12}$. The integral of any function $f$ over a $j$-th sub-triangle's surface $A_{j}$ can be approximated as:

$$
\iint_{A_{j}} f(\alpha, \beta, \gamma) d A \approx A_{j} \sum_{i=1}^{N_{S}} w_{i} f\left(\alpha_{i}, \beta_{i}, \gamma_{i}\right),
$$


in which $\alpha, \beta$ and $\gamma$ are barycentric coordinates inside the $j$-th triangle, and $w_{i}$ are the weights assigned to each quadrature point $i$.

To calculate both forces and moments caused by a specific pressure/traction in a triangle, the coordinates of the integration test points are determined first. Using Eq. (6) the surface integral for the normal contact force on the intersection polygon $S_{12}$ is approximated:

$$
\mathbf{F}_{n}^{S}=\sum_{j=1}^{N_{S}} \sum_{i=1}^{N_{Q}} A_{j} w_{j, i} p_{n}\left(\mathbf{x}_{j, i}\right) \hat{\mathbf{n}}_{12}
$$

where $p_{n}\left(\mathbf{x}_{j, i}\right)$ is the normal contact pressure according to Eq. (4). $N_{Q}$ is the number of quadrature points and $N_{S}$ is the number of sub-triangles in $S$. Additionally, the moments generated by each evaluated pressure with respect to the contact point $\mathbf{c}$ are summed (see also [19]):

$$
\mathbf{M}_{n}^{S}=\sum_{j=1}^{N_{S}} \sum_{i=1}^{N_{Q}} A_{j} w_{j, i} p_{n}\left(\mathbf{x}_{j, i}\right)\left[\left(\mathbf{x}_{j, i}-\mathbf{c}\right) \times \hat{\mathbf{n}}_{12}\right] .
$$

\subsection{Tangential forces}

The tangential relative contact velocity $\mathbf{v}_{12}^{t}$ at $\mathbf{x}$ is calculated as:

$$
\mathbf{v}_{12}^{t}(\mathbf{x})=\mathbf{v}_{12}(\mathbf{x})-\left(\mathbf{v}_{12}(\mathbf{x}) \cdot \hat{\mathbf{n}}_{12}\right) \hat{\mathbf{n}}_{12} .
$$

A simple two-parameter Coulomb Friction model is used with a viscous damper in the static regime. For a given test point $\mathbf{x}$, the pressure due to friction is expressed as:

$$
p_{t, d}(\mathbf{x})=-\min \left(c_{t}\left\|\mathbf{v}_{12}^{t}(\mathbf{x})\right\|, \mu\left\|p_{n}(\mathbf{x})\right\|\right)
$$

where $c_{t}$ is a viscous damping constant $\left(\mathrm{kg} /\left(\mathrm{m}^{2} \mathrm{~s}\right)\right), \mu$ is the Coulomb friction coefficient $(-)$ and $p_{n}(\mathbf{x})=p_{n, e}(\mathbf{x})+p_{n, d}(\mathbf{x})$ is the total normal contact pressure in $\mathbf{x}$.

Analogous to the normal forces, the tangential contact forces are obtained by numerically integrating these pressures over the sub-triangles of $S_{i j}$. For a given triangle, the total tangential force becomes:

$$
\mathbf{F}_{t}^{S}=\sum_{j=1}^{N_{S}} \sum_{i=1}^{N_{Q}} A_{j} w_{j, i} p_{t}\left(\mathbf{x}_{j, i}\right) \frac{\mathbf{v}_{12}^{t}\left(\mathbf{x}_{j, i}\right)}{\left\|\mathbf{v}_{12}^{t}\left(\mathbf{x}_{j, i}\right)\right\|}
$$


and the sum of moments with respect to the contact point:

$$
\mathbf{M}_{t}^{S}=\sum_{j=1}^{N_{S}} \sum_{i=1}^{N_{Q}} A_{j} w_{j, i} p_{t}\left(\mathbf{x}_{j, i}\right)\left[\left(\mathbf{x}_{j, i}-\mathbf{c}\right) \times \frac{\mathbf{v}_{12}^{t}\left(\mathbf{x}_{j, i}\right)}{\left\|\mathbf{v}_{12}^{t}\left(\mathbf{x}_{j, i}\right)\right\|}\right]
$$

\subsection{Transfer of forces and moments to the rigid body}

The triangle forces $\mathbf{F}_{n}^{S}$ and $\mathbf{F}_{t}^{S}$ can be directly summed up to the center of mass of the triangle's parent body. The rigid body moment is the sum of the triangle's moment and the moment of the triangle forces with respect to the contact point c. For polygon $P_{1}$ :

$$
\begin{aligned}
\mathbf{F}_{1}^{\text {dof }} & =-\mathbf{F}_{n}^{S}-\mathbf{F}_{t}^{S}, \\
\mathbf{M}_{1}^{\text {dof }} & =-\mathbf{M}_{t}^{S}-\mathbf{M}_{n}^{S}-\left(\mathbf{c}-\mathbf{x}_{1}^{\text {dof }}\right) \times\left(\mathbf{F}_{t}^{S}+\mathbf{F}_{n}^{S}\right),
\end{aligned}
$$

and for $P_{2}$ :

$$
\begin{aligned}
\mathbf{F}_{2}^{\text {dof }} & =\mathbf{F}_{n}^{S}+\mathbf{F}_{t}^{S}, \\
\mathbf{M}_{2}^{\text {dof }} & =\mathbf{M}_{t}^{S}+\mathbf{M}_{n}^{S}+\left(\mathbf{c}-\mathbf{x}_{2}^{\text {dof }}\right) \times\left(\mathbf{F}_{t}^{S}+\mathbf{F}_{n}^{S}\right),
\end{aligned}
$$

\subsection{Equation of motion}

The Euler equations of motion for each body $p$ with position vector $\mathbf{r}_{p}^{\text {dof }}$ and angular velocity $\mathbf{w}_{p}^{\text {dof }}$ can be written as:

$$
\begin{aligned}
m_{p} \frac{\partial^{2} \mathbf{r}_{p}^{\text {dof }}}{\partial t^{2}} & =\sum \mathbf{F}_{p}^{\text {dof }} \\
\hat{\mathbf{I}}_{p} \frac{\partial \mathbf{w}_{p}^{\text {dof }}}{\partial t}+\mathbf{w}_{p}^{\text {dof }} \times\left(\hat{\mathbf{I}}_{p} \mathbf{w}_{p}^{\text {dof }}\right) & =\sum \mathbf{M}_{p}^{\text {dof }}
\end{aligned}
$$

in which $\hat{\mathbf{I}}_{p}$ is the tensorial moment of inertia in the inertial frame. A leapfrog scheme is used to integrate these equations of motion.

\section{Results and Discussion}

In DEM simulations, the objective is generally not to capture individual force-indentation behaviour during collision events in a realistic way. Instead, the aim is to correctly describe the momentum and energy changes of individual collisions in order to simulate the collective dynamics of a particle system. 
It is common for simulations to artificially reduce the effective stiffness of particles in order to enable larger time steps and hence a bigger simulation time [28, 29, 30]. Damping coefficients are then changed accordingly, to ensure that the energy exchange during collisions remains unchanged.

In this work, the layer stiffness $k_{l}$ is chosen large enough to only allow for a very small overlap between the particles, but low enough to use a reasonably high timestep. Wachs et al. [30] suggest to fix the particles' stiffness based on a maximally allowed overlap distance, which should be small relative to the particle size. The normal damping coefficient is subsequently calculated based on $k_{l}$ and the measured coefficient of restitution $e_{n}$, which, for a given collision, is the ratio between the magnitude of the relative velocity before and after the collision.

\subsection{Validation simulations}

To validate the performed method, we compare our simulations to an experimental study by Latham et al. [31, 32], as well as computational results using another method by Wachs et al. [30]. In the first study, 648 wooden cubes were deposited into a rectangular box $(250 \times 250 \times 375 \mathrm{~mm})$ in a fixed snake-like sequence. With a measured Coulomb friction coefficient $\mu$ of 0.5 , the authors estimated an average porosity of $33 \%$.

\begin{tabular}{llll}
\hline Parameter & Value [32] & Value [30] & Units \\
\hline timestep & $4 \cdot 10^{-5}$ & $1 \cdot 10^{-5}$ & $\mathrm{~s}$ \\
simulation time & 35.4 & 10.33 & $\mathrm{~s}$ \\
cube side length & 0.024177 & 0.00643 & $\mathrm{~m}$ \\
cube mass & 0.00923 & 0.00031 & $\mathrm{~kg}$ \\
cube density & 653 & 1163.66 & $\mathrm{~kg} / \mathrm{m}^{3}$ \\
layer stiffness $\left(k_{l}\right)$ & $30 \cdot 10^{6}$ & $80 \cdot 10^{6}$ & $\mathrm{~Pa} / \mathrm{m}$ \\
coefficient of restitution $\left(e_{n}\right)$ & 0.25 & 0.85 & - \\
tangential friction coefficient $(\mu)$ & 0.5 & 0.5 & - \\
tangential dashpot constant $\left(c_{t}\right)$ & $4 \cdot 10^{6}$ & $4 \cdot 10^{6}$ & $\mathrm{~kg} /\left(\mathrm{m}^{2} \mathrm{~s}\right)$ \\
number of cubes & 648 & 250 & - \\
\hline
\end{tabular}

Table 1: Simulation parameters and mechanical parameters used in validation simulation of gravitational cube deposition for Latham et al. 32] and Wachs et al. 30. simulations (see Fig. 2)

In Fig. 2(a), the cube deposition process is visualized. Similar to [32], a horizontal plane is shown, which corresponds to the height the cubes would reach if they had the experimentally estimated porosity of $33 \%$, and is purely 
a function of the number of cubes in the simulation. As can be visually inspected, the final height of the plane corresponds very well to maximal heights of the cubes. The simulation parameters, listed in Table 1, correspond to the experimental settings, only with the cubes' stiffness artificially lowered as explained above. A movie of this simulation can be found in the supplementary information. In a second validation simulation, the packing of cubes in a cylindrical container with internal diameter $50 \mathrm{~mm}$ and height $130 \mathrm{~mm}$ is compared to the simulation results by [30]. There, a porosity of $43.4 \%$ was reported for 250 cubes with a friction coefficient of 0.5 (simulation parameters in Table 1). In Fig. 2(b), the simulated deposition sequence is visualized, with the horizontal plane corresponding to a porosity of $42 \%$.

\subsection{Packing densities of different convex geometries}

In order to show the applicability of the model for arbitrary shapes, as well as to investigate the effect of shape on porosity in gravitational deposition, additional simulations were performed for various shapes. First, we consider shapes with triangular facets: tetrahedra, icosahedra, and two levels of subdivided icosahedra (see also [19]). Apart from the particles' shape, the simulation parameters are identical to the cube deposition as performed by Latham et al. [32], i.e. they have the same volume, mass, and mechanical contact properties. The packing of these shapes is compared to spheres and cubes. For each shape, three different simulation runs are performed - using different initial random orientations of the particles. In the supplementary information, movies can be found of the gravitational deposition of spheres and tetrahedra.

In order to make a valid comparison with spheres, the contact model for spheres cannot be considered Hertzian, as the integrated contact force given in Eq. (7), would result in a different force-overlap relationship. Instead, it can be shown that for the contact of a sphere with radius $R$, with a surface consisting of infinitely small facets, the force-indentation relationship would be the following:

$$
\mathbf{F}_{n}=\pi k_{l} \delta_{12}^{2}\left(R-\frac{\delta_{12}}{2}\right) \hat{\mathbf{n}}_{12}
$$

whereas Hertz' force would yield a $F \sim \delta_{12}^{3 / 2}$ relation. Since this contact force increases as $\sim \delta_{12}^{2}$ for small indentations $\left(R \gg \delta_{12}\right)$, Eq. (19) has been used to perform the simulations with spheres. This guarantees that discrepancies in packing are truly due the particle's shape and not artifacts due to a 
slightly different contact force law. It was found that, as long as the particle stiffness is large enough, the final packing density when using Hertz' law does not differ significantly compared to when using Eq. (19).

Fig. 3 shows the results of gravitational deposition for spheres, tetrahedra and icosahedra in a rectangular box. The horizontal plane indicates a porosity height of $33 \%$, similar to Fig. 2. In order to quantify packing density more in detail, a packing factor $P$ is calculated as following [19]:

$$
P=\frac{N_{h} V_{p}}{A_{b} h} 100
$$

with $h$ the height of a horizontal plane submerged in the particle stacking, $N_{h}$, the number of particles with a center of mass under $h, V_{p}$ the volume of one particle and $A_{b}$ the area of the bottom of the box $(250 \times 250 \mathrm{~mm})$. It should be noted that, when $h$ is chosen well within the particle stacking, this measure for the packing factor disregards the loosely packed particles in the upper layers, and hence gives rise to higher packing densities than an average packing density calculated from all particles. On the other hand, because this measure is less influenced by outliers in the top layers, its value is more constant over multiple simulation runs. In this work, an average $\hat{P}$ was calculated as the mean packing factor for 100 planes chosen at $h$ between 140 and $180 \mathrm{~mm}$.

Fig. 4 shows values and standard deviations of $\hat{P}$ for three simulation runs, indicating significant differences with sphere and cube packings. Interestingly, whereas tetrahedra pack significantly worse than spheres, icosahedra pack significantly better. When subdividing the icosahedron (see [19, 33], subdivided nodes are projected on the sphere surface), one or two times, the packings become identical to the sphere packing. All the triangulated shapes pack significantly worse than cubes. It should be pointed out that these results cannot necessarily be generalized to different shapes and relative sizes of the container.

It is not surprising that the packing density from gravitational deposition for cubes is higher than for spheres, tetrahedra, or icosahedra, as its theoretical maximal packing density in a rectangular container is much higher. To investigate this further, we performed simulations of gravitational deposition of non-cubical objects with rectangular, orthogonal facets. For this, shapes are introduced which are used in the game "Tetris". These simulations serve to investigate packing factors from gravitational deposition for objects with very high maximal theoretical packing densities, as well as to demonstrate 
the applicability of the model to non-convex shaped bodies.

\subsection{Packing of shapes with concavities}

Again, the volume and mass of the shapes is chosen to correspond with the cubes as described by Latham et al. 32. Simulations are performed with squares, I-shapes, L-shapes, Z-shapes, and a mixture of these five. Fig. 5 shows the final packings of these shapes after gravitational deposition in a rectangular box. Again, the horizontal plane corresponds to a porosity of $33 \%$. The mean packing factors $\hat{P}$ - see Eq. 20 - are summarized in Fig. 4 . Movies of these simulations can be found in the supplementary information. All these shapes pack significantly worse than cubes. Moreover, a clear distinction can be made between the purely convex rectangular shapes (I-shapes and squares), which yield higher packing densities than spheres, and shapes also containing concave regions (Z-shapes, L-shapes and T-shapes) which pack significantly worse than spheres. Although these shapes could theoretically result in very high packing densities in a rectangular container, their concave regions create shielded empty spaces during gravitational deposition, which cannot be filled without fluidizing a large region of particles.

Interestingly, the mixture of all shapes packs better than the concaveshaped particles alone. We hypothesized that a linear combination of the packings densities of each shape, weighted by their fraction in the mixture, could be used as a predictor for the mixture packing factor. However, this would predict a significantly higher packing density. An explanation for this discrepancy could be that the chance of alignment of the rectangular shaped bodies is sharply reduced by the disorder caused by the concaveshaped particles.

\subsection{Computational performance}

In order to assess the computational performance of the proposed method a simulation was constructed of particles in a rotating cylinder $(\varnothing=40$ $\mathrm{cm}$, angular velocity $\omega=2 \mathrm{~s}^{-1}$ ). This set-up was chosen because the sys-

tem evolves to a steady-state flow in which the average forces, velocity and number of contacts remains conserved. We compare between spheres, cubes and a detailed triangulated mesh of a nut-shape composed out of 444 triangles/particle (see Fig. 6). The total number of particles was varied, and the length of the cylinder adapted to roughly conserve the filling height in the 
cylinder between different particle numbers. Times are reported for simulating stead-state flow during 8000 time steps (timestep: $4 \times 10^{-5} \mathrm{~s}$ ). Simulations were run single-threaded on a desktop CPU (AMD opteron 6370).

\begin{tabular}{llll}
\hline Shape & Spheres & Cubes & Nuts \\
Time $(\mathrm{s})$ & 42.29 & $1.43 \cdot 10^{3}$ & $1.23 \cdot 10^{4}$ \\
\hline
\end{tabular}

Table 2: Total computational time (s) to simulate 3000 spheres, cubes and nuts for 8000 time steps in a rotating drum (see Fig 6) on a desktop CPU (AMD Opteron 6378 Processor).

Table 2 reports the times for systems of 3000 particles. As the particle shape gets more complex, the computational times greatly increase. Fig. 7 shows the scaling of the computational cost with the number of contact primitives $N_{\text {prim. }}$. For all three shapes, the computational time scales quasilinearly with $N_{\text {prim }}$. For simple polyhedral shapes, for which the facets span the complete size of the particle, like cubes, the average cost per primitive is higher than for spheres. Indeed, a typical cube-cube collision involves at least four intersecting squares (corner-plane contact). Moreover, the average computational effort per primitive is higher due to the more complicated geometrical calculation (e.g. Eq.77and 8). Interestingly, however, the calculation time per contact primitive decreases for the more complicated particles. As overlap distances in DEM are typically very small, the number of contacting primitives between two particles only slightly increases when the particle mesh gets more refined. This last point emphasizes the strength of the proposed method. An optimized grid-based contact detection algorithm ensures that only nearly colliding primitives are selected as contact candidates. As illustrated, the simulation of a large number of highly detailed particles is feasible within a reasonable computation time. A study on the computational performance of a similar approach but for rounded bodies can be found in [19].

\section{Conclusion and Outlook}

In this study, a novel method was presented for simulating arbitrarilyshaped particles consisting of polygonal facets in the Discrete Element Method. Two bodies in contact are simulated as a set of interacting polygon-shaped contact primitives. As these primitives only need to contain local information about the geometry and mechanical properties, the method provides a very 
flexible framework to simulate contact interactions between particles of any shape and potentially non-uniform mechanical properties. Since there is no need for determining a unique contact point and normal unit vector for the contact between two arbitrary shapes, the method is not restricted to convex bodies and does not require disassembling arbitary shapes into sets of convex bodies. It was shown that the computational cost scales quasi linearly with the number of contact primitives / particles and that - although introducing a clear additional overhead for "simple" shapes - the relative computational efficiency scales favorably when the particle shape becomes more complex. Furthermore, because each polygon-polygon contact can be individually resolved without information of the surrounding primitives, the method lends itself very well for parallelization.

The presented method has been validated by comparing to the gravitational deposition of cubes experimentally measured and simulated by Latham et al. 31, 32] and Wachs et al. [30]. After replicating the measured porosity for cubes, we calculated packing factors from simulations of gravitational deposition for various other particles shapes. Moreover, it was verified that the packing density converges to the solution for exact spheres when the polyhedral shapes approaches the sphere.

Conclusions on these packing densities cannot be drawn independent of the (rectangular) shape of the container. Nonetheless, both spheres and tetrahedra have been shown to pack significantly worse than beams. For beams, the packing factor decreases with increasing aspect ratio. It is well known that porosity in packings increases with particle elongation [34]. This has been attributed to the growth of the orientation average excluded volume [35]. When composites of multiple beams contain concave regions, the packing factor is strongly reduced.

In the future this method could be used to investigate the effects mixtures of different particle shapes have on the geometry and mechanics of packings, heaps and on particle flow behavior. As previous research has pointed out [36, 37, 3, 14], approximating particles in granular materials as spheres is often insufficient to predict stability and dynamics of flow. Having a robust and efficient method available to account for arbitrary particle shapes will help to better simulate and eventually better understand these systems. 


\section{Acknowledgements}

This research was funded by the Agency for Innovation by Science and Technology in Flanders (IWT), grant nr. 111504.

\section{References}

[1] C. Nouguier-Lehon, B. Cambou, E. Vincens, Influence of particle shape and angularity on the behaviour of granular materials: a numerical analysis, International Journal for Numerical and Analytical Methods in Geomechanics 27 (14) (2003) 1207-1226.

[2] A. Pena, R. Garcia-Rojo, H. Herrmann, Influence of particle shape on sheared dense granular media, Granular Matter 9 (3-4) (2007) 279-291.

[3] G.-C. Cho, J. Dodds, J. C. Santamarina, Particle shape effects on packing density, stiffness, and strength: natural and crushed sands, Journal of Geotechnical and Geoenvironmental Engineering 132 (5) (2006) 591602 .

[4] F. Alonso-Marroquin, H. Herrmann, Ratcheting of granular materials, Physical review letters 92 (5) (2004) 054301.

[5] T. Pöschel, T. Schwager, Computational Granular Dynamics, Springer, 2005.

[6] L. Vu-Quoc, X. Zhang, O. Walton, A 3-d discrete-element method for dry granular flows of ellipsoidal particles, Computer methods in applied mechanics and engineering 187 (3) (2000) 483-528. doi:10.1016/ S0045-7825 (99)00337-0.

[7] Q. Zheng, Z. Zhou, A. Yu, Contact forces between viscoelastic ellipsoidal particles, Powder Technology 248 (2013) 25-33. doi:10.1016/j . powtec.2013.03.020.

[8] B. Yan, R. A. Regueiro, S. Sture, Three-dimensional ellipsoidal discrete element modeling of granular materials and its coupling with finite element facets, Engineering Computations 27 (4) (2010) 519-550. 
[9] D. S. Preece, R. P. Jensen, E. D. Perkins, J. R. Williams, Sand production modeling using superquadric discrete elements and coupling of fluid flow and particle motion, Tech. rep., Sandia National Labs, Albuquerque, NM (US); (1999).

[10] C. Wellmann, P. Wriggers, A two-scale model of granular materials, Computer Methods in Applied Mechanics and Engineering 205208 (0) (2012) 46 - 58, special Issue on Advances in Computational Methods in Contact Mechanics dedicated to the memory of Professor J.A.C. Martins. doi:http://dx.doi.org/10.1016/j.cma.2010.12.023.

URL http://www.sciencedirect.com/science/article/pii/ S0045782510003798

[11] Y. Feng, K. Han, D. Owen, Energy-conserving contact interaction models for arbitrarily shaped discrete elements, Computer Methods in Applied Mechanics and Engineering 205208 (0) (2012) 169 - 177, special Issue on Advances in Computational Methods in Contact Mechanics dedicated to the memory of Professor J.A.C. Martins. doi:http://dx.doi.org/10.1016/j.cma.2011.02.010. URL http://www.sciencedirect.com/science/article/pii/ S0045782511000454

[12] J. Favier, M. Abbaspour-Fard, M. Kremmer, A. Raji, Shape representation of axi-symmetrical, non-spherical particles in discrete element simulation using multi-element model particles, Engineering Computations 16 (4) (1999) 467-480. doi:10.1108/02644409910271894.

[13] J.-F. Ferellec, G. R. McDowell, A method to model realistic particle shape and inertia in dem, Granular Matter 12 (5) (2010) 459-467. URL http://link.springer.com/article/10.1007/ s10035-010-0205-8

[14] M. Lu, G. McDowell, The importance of modelling ballast particle shape in the discrete element method, Granular Matter 9 (2007) 69-80.

[15] J. F. Peters, M. A. Hopkins, R. Kala, R. E. Wahl, A poly-ellipsoid particle for non-spherical discrete element method, Engineering Computations 26 (6) (2009) 645-657.

URL http://www.emeraldinsight.com/journals.htm?articleid= 1806108\&amp; show=abstract 
[16] L. Pournin, T. M. Liebling, A generalization of distinct element method to tridimensional particles with complex shapes, P\&G05 2 (2005) 13751378 .

[17] J. E. Andrade, K.-W. Lim, C. F. Avila, I. Vlahinić, Granular element method for computational particle mechanics, Computer Methods in Applied Mechanics and Engineering 241 (2012) 262-274.

[18] K.-W. Lim, J. E. Andrade, Granular element method for threedimensional discrete element calculations, International Journal for Numerical and Analytical Methods in Geomechanics 38 (2) (2014) 167-188.

[19] B. Smeets, T. Odenthal, J. Keresztes, S. Vanmaercke, P. Van Liedekerke, E. Tijskens, W. Saeys, H. Van Oosterwyck, H. Ramon, Modeling contact interactions between triangulated rounded bodies for the discrete element method, Computer Methods in Applied Mechanics and Engineering 277 (2014) 219-238.

[20] M. C. Lin, D. Manocha, J. Cohen, Collision detection: Algorithms and applications.

[21] A. Garcia-Alonso, N. Serrano, J. Flaquer, Solving the collision detection problem, Computer Graphics and Applications, IEEE 14 (3) (1994) 3643.

[22] M. Held, J. T. Klosowski, J. S. Mitchell, Evaluation of collision detection methods for virtual reality fly-throughs, in: Canadian Conference on Computational Geometry, Citeseer, 1995, pp. 205-210.

[23] A. Munjiza, E. Rougier, N. W. M. John, Mr linear contact detection algorithm, International Journal for Numerical Methods in Engineering 66 (1) (2006) 46-71. doi:10.1002/nme.1538.

[24] K. He, S. Dong, Z. Zhou, Multigrid contact detection method, Phys. Rev. E 75 (3) (2007) 036710.

[25] M. Moore, J. Wilhelms, Collision detection and response for computer animation, in: ACM Siggraph Computer Graphics, Vol. 22, ACM, 1988, pp. 289-298. 
[26] H. Noborio, S. Fukuda, S. Arimoto, Fast interference check method using octree representation, Advanced robotics 3 (3) (1988) 193-212. doi: 10.1163/156855389X00091.

[27] G. Hippmann, An algorithm for compliant contact between complexly shaped surfaces in multibody dynamics, Multibody Dynamics, Jorge AC Ambrosio (Ed.), IDMEC/IST, Lisbon, Portugal, July 14.

[28] P. W. Cleary, Large scale industrial dem modelling, Engineering Computations 21 (2/3/4) (2004) 169-204.

[29] P. W. Cleary, Dem prediction of industrial and geophysical particle flows, Particuology 8 (2) (2010) 106-118.

[30] A. Wachs, L. Girolami, G. Vinay, G. Ferrer, Grains3d, a flexible dem approach for particles of arbitrary convex shapepart i: numerical model and validations, Powder Technology 224 (2012) 374-389.

[31] A. Munjiza, J. Latham, Comparison of experimental and fem/dem results for gravitational deposition of identical cubes, Engineering Computations $21(2 / 3 / 4)(2004)$ 249-264.

[32] J. Latham, A. Munjiza, The modelling of particle systems with real shapes, Philosophical Transactions of the Royal Society of London Series A Mathematical Physical and Engineering Sciences 362 (2004) 19531972 .

[33] T. Odenthal, B. Smeets, P. Van Liedekerke, E. Tijskens, H. Van Oosterwyck, H. Ramon, Analysis of initial cell spreading using mechanistic contact formulations for a deformable cell model, PLoS Computational Biology 9 (10) (2013) e1003267. doi:10.1371/journal.pcbi.1003267.

[34] R. Zou, A. Yu, Evaluation of the packing characteristics of mono-sized non-spherical particles, Powder technology 88 (1) (1996) 71-79.

[35] A. V. Kyrylyuk, A. P. Philipse, Effect of particle shape on the random packing density of amorphous solids, physica status solidi (a) 208 (10) (2011) 2299-2302. 
[36] A. K. Ashmawy, B. Sukumaran, V. V. Hoang, Evaluating the influence of particle shape on liquefaction behavior using discrete element modeling, in: Proc. Offshore and Polar Engineering Conference, 2003, pp. $542-550$.

[37] J. Santamarina, G. Cho, Soil behaviour: The role of particle shape, in: Advances in geotechnical engineering: The skempton conference, Vol. 1, Thomas Telford, 2004, pp. 604-617. 


\section{Numerical considerations for contact resolution}

To avoid calculating unnecessary contacts, as well as to ensure sufficient accuracy in almost flat contacts, the implementations includes a few additional calculations, which are discussed in this section.

\section{Contact rejection cases}

A few simple checks can be performed to reject potential contact candidates and therefore avoid unnecessary calculations. A first requirement for two contacting polygons is that their normals face towards each other. For this the following rejection criterion is employed:

$$
\hat{\mathbf{n}}_{1} \cdot \hat{\mathbf{n}}_{2}>0 \text {. }
$$

Without additional information about the shape of the complete body, it is impossible to distinguish two valid contact candidates from contact candidates on opposite sides of two shapes (see Fig. 8). This is resolved by also taking into account the local layer thicknesses $h_{1}$ and $h_{2}$. For each polygon these are defined as the cross diameter of the body normal to the polygon and can be (pre-)computed by shooting a ray from the center of a given polygon in the direction opposite to its outward normal vector. The distance between the first polygon intersection of this ray and the center of the polygon determines the layer thickness.

Let $\mathbf{x}_{i}^{c}$ be the center point of polygon $P_{i}$ with layer thickness $h_{i}$. The interior point $\mathbf{x}_{i}^{\mathrm{s}}$ (see also Fig. 8) is calculated as:

$$
\mathbf{x}_{i}^{\mathrm{s}}=\mathbf{x}_{i}^{c}-\frac{1}{2} h_{i} \hat{\mathbf{n}}_{i}
$$

For two polygons $P_{1}$ and $P_{2}$, the rejection criterion becomes:

$$
\left(\mathbf{x}_{1}^{\mathrm{s}}-\mathbf{x}_{2}^{\mathrm{s}}\right) \cdot \hat{\mathbf{n}}_{12}<\left|\left(\mathbf{x}_{1}^{\mathrm{c}}-\mathbf{x}_{2}^{\mathrm{c}}\right) \cdot \hat{\mathbf{n}}_{12}\right|
$$

The implication of this rejection criterion is that this method does not allow for indentations which are bigger than $\min \left(0.5 h_{1}, 0.5 h_{2}\right)$. For relatively stiff particles this is generally not a concern in DEM simulations, but this might pose a constraint for simulating very thin (sheet-like) particle shapes.

A trivial third criterion is used when $S_{i j}$ has been computed: $P_{1}$ and $P_{2}$ have no intersection area where the overlap distance would be positive:

$$
S_{i j}=\emptyset
$$


Approximate flat contacts

When $P_{1}$ and $P_{2}$ are almost exactly parallel, their planes' intersection line $\hat{\mathbf{l}}_{i j}$ cannot be calculated correctly and the contact calculations can be greatly simplified. The criterion used for two parallel planes is:

$$
\left\|\hat{\mathbf{n}}_{1} \times \hat{\mathbf{n}}_{2}\right\|<\sin \left(\alpha_{\max }\right)
$$

with $\alpha_{\max }$ the maximum angle allowed between the two planes for which they are still considered parallel. In this study, $\alpha_{\max }$ was chosen at $0.001^{\circ}$.

If $P_{1}$ and $P_{2}$ are parallel, $S_{12}$ is simply the complete intersection between their projections: $P_{1}^{\prime}$ and $P_{2}^{\prime}$. The contact point is the center point of $S_{12}$, and the overlap distance $\delta_{12}$ is calculated as:

$$
\delta_{12}=\left(\mathbf{x}_{1}^{\mathrm{c}}-\mathbf{x}_{2}^{\mathrm{c}}\right) \cdot \hat{\mathbf{n}}_{12}
$$




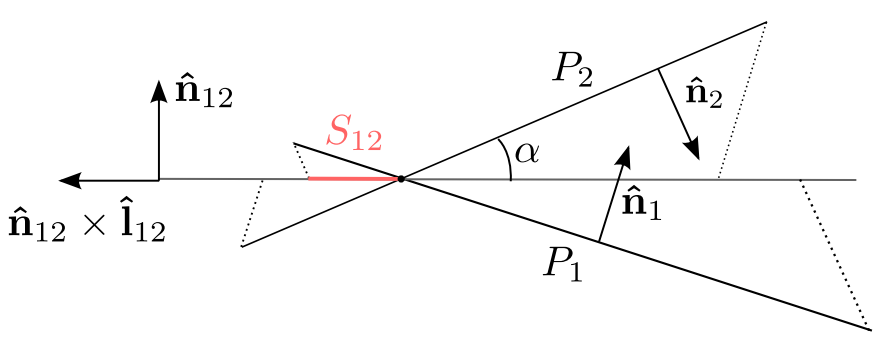

(a) Side view

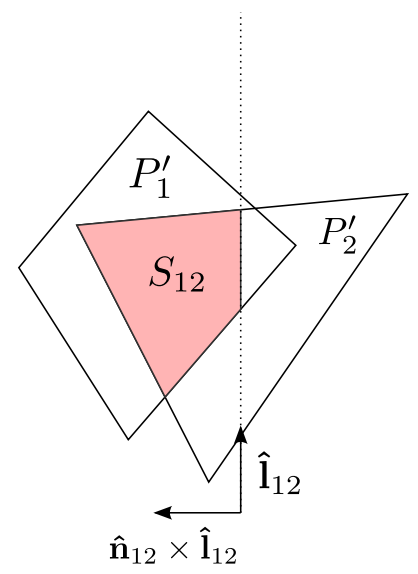

(b) Top view

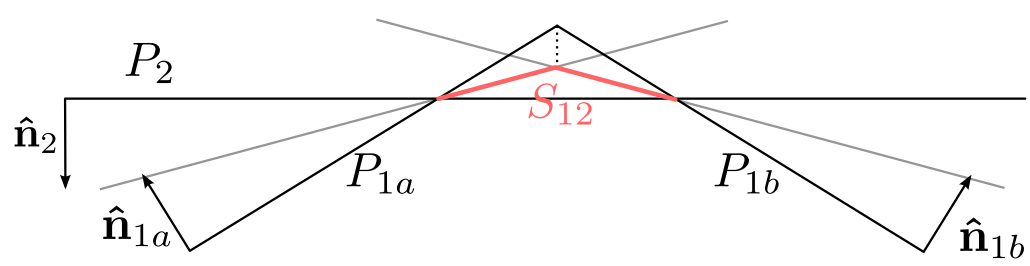

(c) Corner contact

Figure 1: (a) Projection of polygon $P_{1}$ and polygon $P_{2}$ onto the contact plane according to the normal of the other polygon, yielding $P_{1}^{\prime}$ and $P_{2}^{\prime}$. (b) Calculation of $S_{12}$ as the intersection of projected polygons $P_{1}^{\prime}$ and $P_{2}^{\prime}$, cut off by the plane-plane intersection line s, $\hat{\mathbf{l}}_{12}$. (c) Contact of a corner of body 1 (polygons $P_{1 a}$ and $P_{1 b}$ ) with $P_{2}$. By projecting $P_{1 a}$ and $P_{1 b}$ along $\hat{\mathbf{n}}_{2}, S_{12}$ is continuous and its corresponding overlap volume is equal to the volume of the indenting corner. 

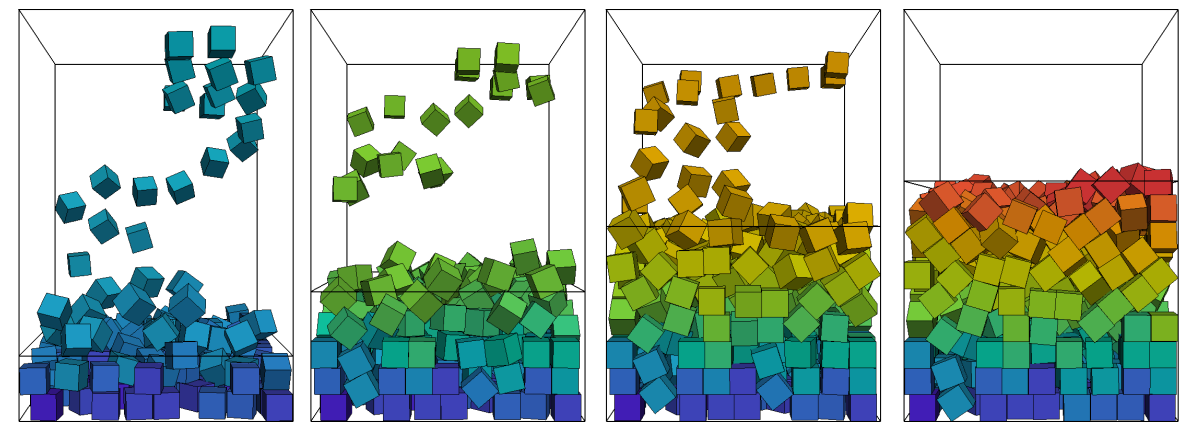

(a)
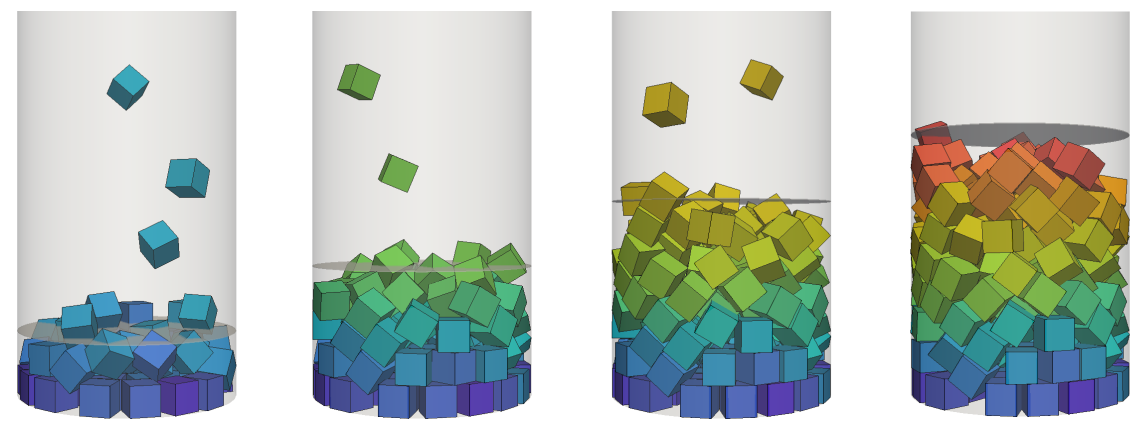

(b)

Figure 2: Simulated deposition of cubes with friction coefficient of 0.5. (a) Deposition based on experiment of Latham et al. [32] in a rectangular container. The volume under the horizontal plane represents a porosity of $33 \%$. From left to right: 162, 324, 486 and 648 cubes. (b) Deposition in a cylindrical container described by Wachs et al. 30]. The volume under the horizontal plane represents a porosity of $42 \%$. From left to right: 62 , 124, 186 and 250 cubes. 

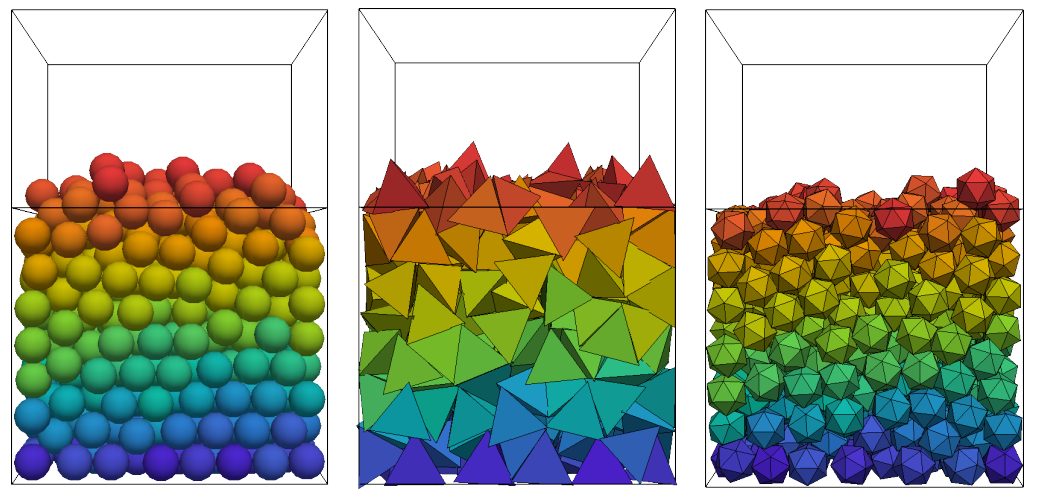

Figure 3: Simulated gravitational deposition of (from left to right) spheres, tetrahedra and icosahedra. The plane indicates the level of $33 \%$ porosity as indicated in Fig. 2, All shapes have identical volume and mechanical properties as the cubes, described in Table 1. 


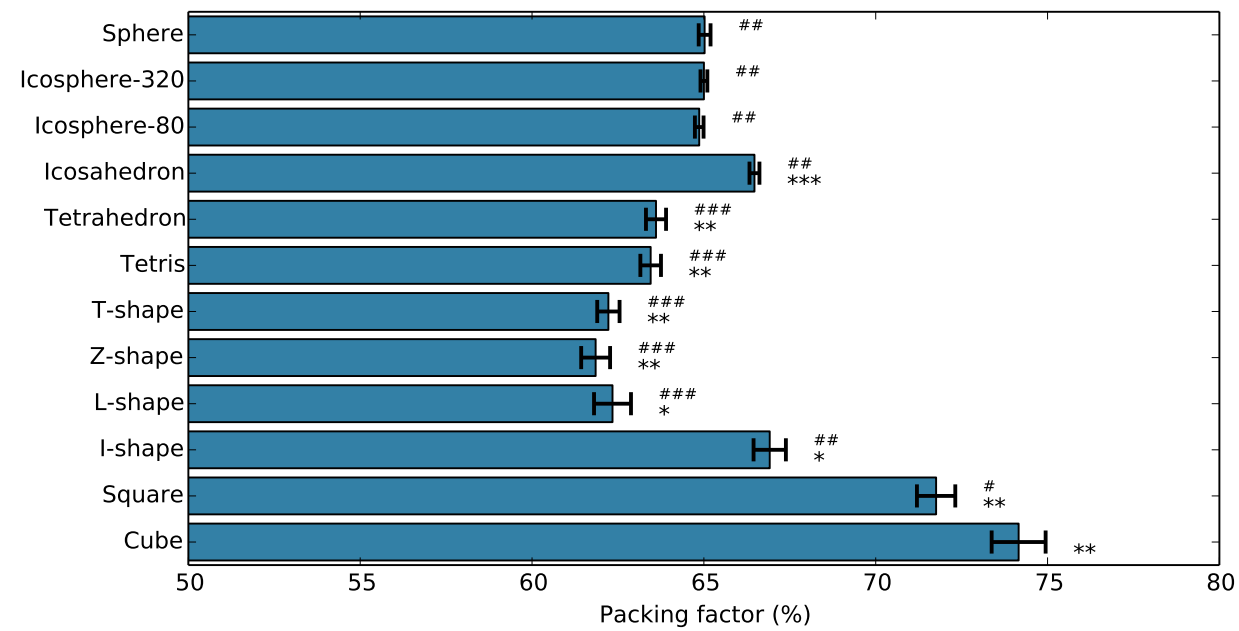

Figure 4: Comparison of packing factors for spheres, tetrahedra, icosahedra, cubes, Lshapes, I-shapes, T-shapes, Z-shapes, square shapes and a mixture of the last five. Stars $(*)$ indicate a significant difference from the sphere packing (p-value: $0.05 * 0.01 * * 0.001$ ***) using a two-sided Welch's t-test. Hashtags $(\#)$ indicate a significant difference from the cube packing (p-value: $\left.0.05{ }^{\#} 0.01{ }^{\# \#} 0.001 \# \# \#\right)$. 

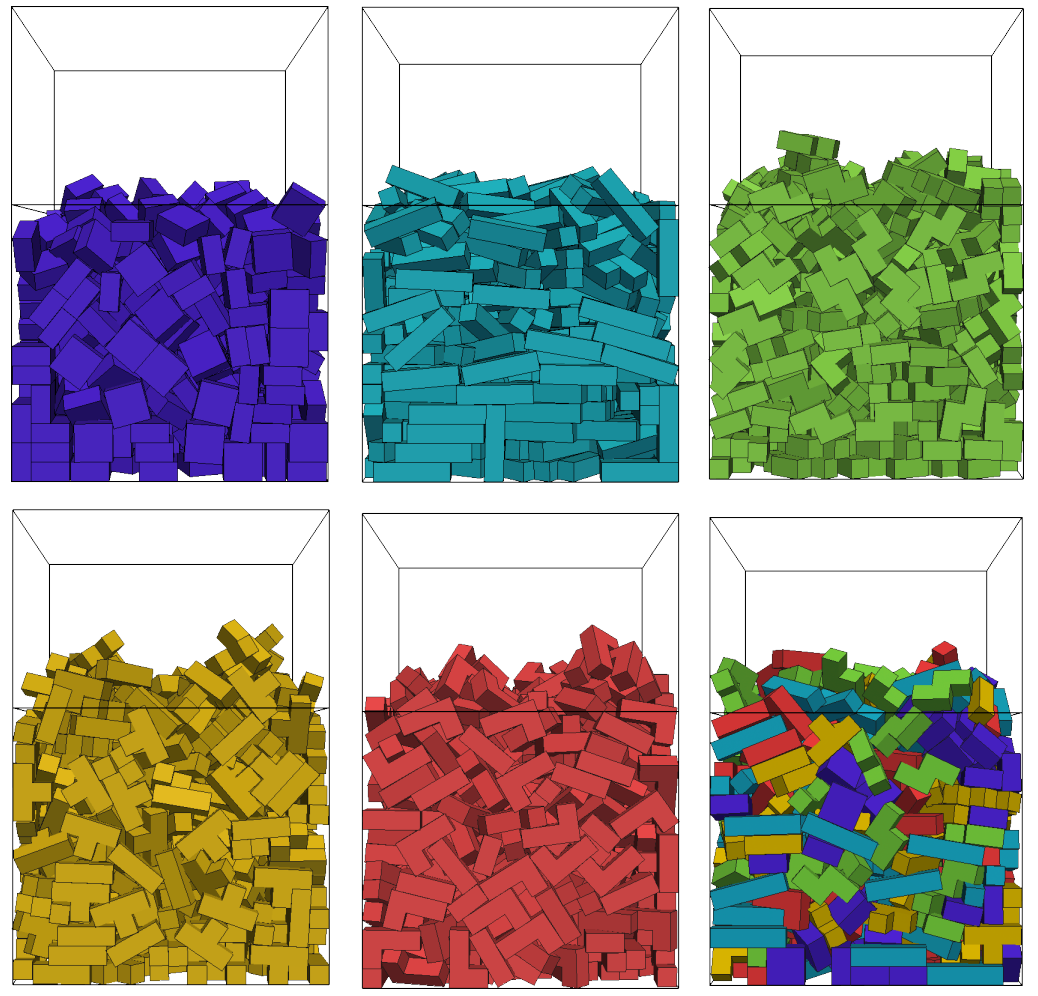

Figure 5: Gravitational deposition of tetris blocks. From left to right, top to bottom: square shapes, I-shapes, Z-shapes,T-shapes, L-shapes and a random mixture of all blocks. The plane indicates the level of $33 \%$ porosity as indicated in Fig. 2. All shapes have identical volume and mechanical properties as the cubes, described in Table1. 


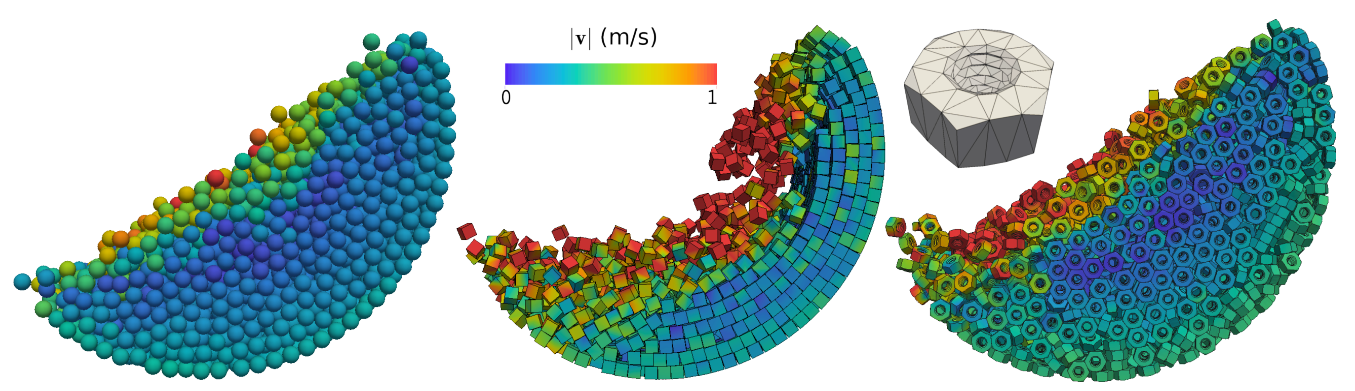

Figure 6: Comparison of simulations of 3000 particles in a rotating drum for spheres (left), cubes (middle) and a complex "nut" mesh (right), with the color scale indicating magnitude of velocity. Snapshots were made at $t=10 \mathrm{~s}$. 


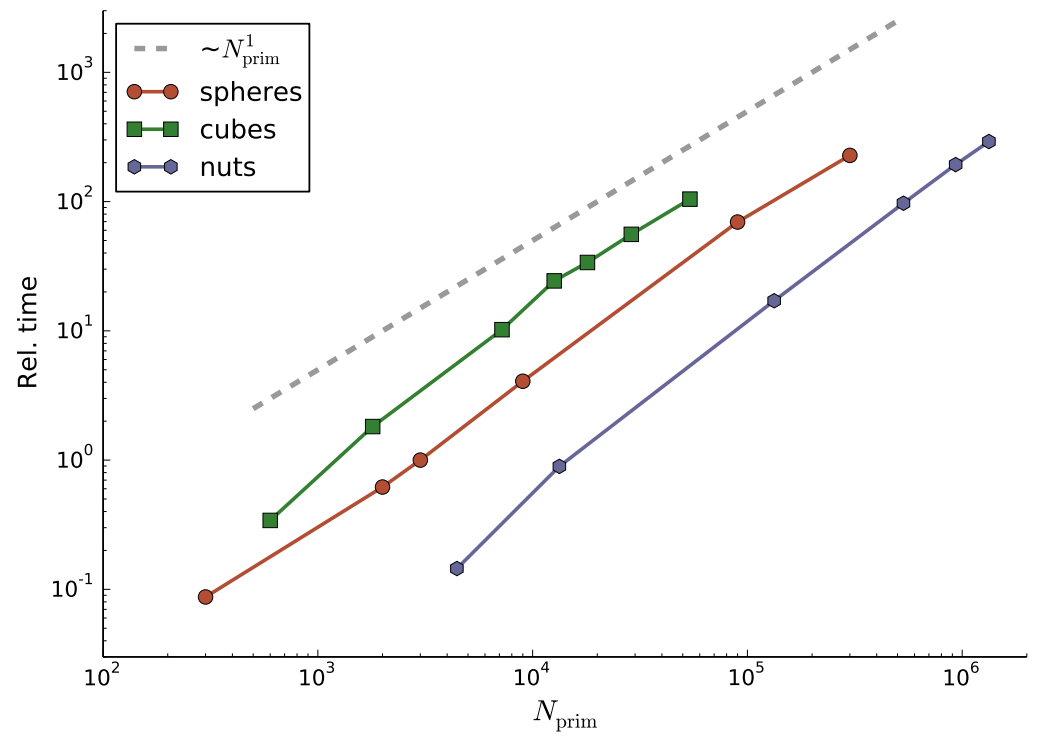

Figure 7: Computational time (relative to a simulation of 3000 spheres) as a function of number of contact primitives for spheres, cubes and nut-shapes. The dashed guide-line shows a linear scaling. 


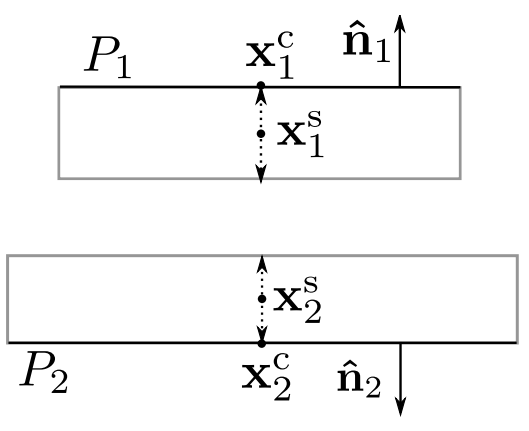

(a) no physical contact

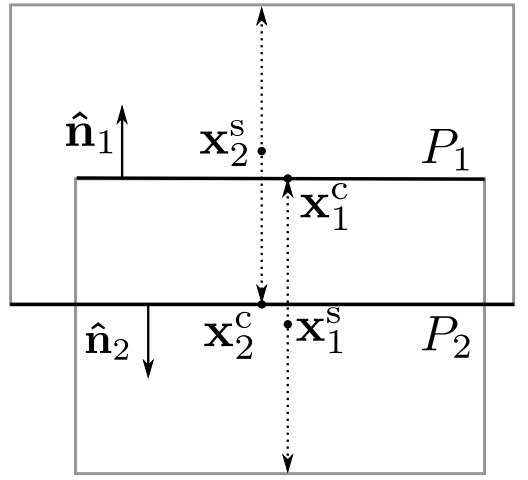

(b) physical contact

Figure 8: (a) $P_{1}$ and $P_{2}$ have opposite normals and would have a non-empty intersection in the contact plane, but this configuration does not represent a physical contact. $\left\|\mathbf{x}_{1}^{\mathrm{s}}-\mathbf{x}_{2}^{\mathrm{s}}\right\|<\left\|\mathbf{x}_{1}^{c}-\mathbf{x}_{2}^{c}\right\|$. (b) $P_{1}$ and $P_{2}$ have opposite normals, a non empty intersection in the contact plane and represent a physical contact condition: $\left\|\mathbf{x}_{1}^{\mathrm{s}}-\mathbf{x}_{2}^{\mathrm{s}}\right\|>\left\|\mathbf{x}_{1}^{c}-\mathbf{x}_{2}^{c}\right\|$. 